\title{
Suppressed demand in the clean development mechanism: conceptual and practical issues
}

\author{
Randall Spalding-Fecher \\ Carbon Limits AS, Norway
}

\begin{abstract}
One of the challenges of applying greenhouse gas emission accounting approaches in poor communities is that the current consumption of many household services (e.g. heating and cooking, lighting and potable water) may not reflect the real demand for those services. This could be the result of lack of infrastructure, lack of natural resources or poverty, particularly the high costs of these services relative to household incomes. The situation of 'suppressed demand' creates a problem for setting emissions baselines against which to compare project performance, and has negatively affected CDM project development in Africa, Least Developed Countries and other regions with very few CDM projects. Ironically, although new large-scale power plants do not have to show that they actually displace other plants (existing or new), many small-scale energy projects can only claim credit for displacing historical (very low level) emissions from households. While the CDM rules are evolving to consider suppressed demand, much more can be done to catalyse investment in these types of climate change mitigation projects in poor communities. Furthermore, making progress will require significant expert and stakeholder input to ensure that simplification is balanced with maintaining overall environmental integrity.

Keywords: Clean development mechanism, climate change mitigation, suppressed demand, energy services, poverty
\end{abstract}

\section{Introduction}

When adopting the Kyoto Protocol in 1997, Parties to the United Nations Framework Convention on Climate Change (UNFCCC) established the clean development mechanism (CDM) with the twin goals of contributing to the sustainable development of developing countries and assisting developed countries to meet their emission limitation targets. This has meant that the rules for the $\mathrm{CDM}$ have had to address not only technical issues around greenhouse accounting but also the how the rules and procedures affect development in host countries. For any project-based mitigation activity under the $\mathrm{CDM}$, the crux of the emissions reduction analysis is determining what would have happened in the absence of the mitigation mechanism or incentive. This includes determining the appropriate 'baseline' for a given project activity (i.e. technology development and emissions without the project activity), as well as the interlinked concept of determining whether the project activity is 'additional' (Ellis et al., 2007; Gillenwater and Seres, 2011; Schneider, 2009).

One of the challenges of applying greenhouse gas (GHG) accounting approaches in poor communities is that the current consumption of many household services (e.g. heating and cooking, lighting and potable water) may not reflect the real demand for those services. This could be the result of a lack of infrastructure, lack of natural resources, or poverty, particularly the high costs of these services relative to household incomes. The situation of 'suppressed demand' creates a problem for setting baselines, because the CDM rules say that the baseline scenario selected for a project should provide the same level of service and quality as the project scenario (Winkler and Thorne, 2002). This is clearly not the case if the project scenario provides a much higher service level, owing to low historical consumption. At the same time, the CDM rules state that, "the baseline may include a scenario where future anthropogenic emissions by sources are projected to rise above current levels, due to the spe- 
cific circumstances of the host Party' (UNFCCC, 2006 para. 46), and in 2009 the Parties to the Kyoto Protocol directed the CDM Executive Board (EB) to, "further explore the possibility of including in baseline and monitoring methodologies, as appropriate, a scenario where future anthropogenic emissions by sources are projected to rise above current levels due to specific circumstances of the host Party' (UNFCCC, 2009). This article examines how suppressed demand has been addressed so far in the CDM system, as well as discussing the need for and implications of increasing the use of suppressed demand in GHG accounting within the CDM.

\section{Background and definitions}

Household energy services for poor non-electrified communities are an important example of the need to consider suppressed demand, because historical energy consumption may not be a good proxy for future energy demand. ${ }^{1}$ Even if we know the energy source used historically (e.g. kerosene for lighting), the quantity of fuel used historically may not represent the actual energy service demand. Energy services include lighting, cooking, space heating and motive power. These are measured not with energy units (kWh or GJ) but in units that reflect the actual service delivered (e.g. lumens of lighting, average indoor temperature, or litres of water heated to a certain temperature) (Modi et al., 2006; Sovacool, 2011). This distinction is very important because, for the same energy consumption and emissions, two different technologies can deliver vastly different energy service levels.

There are two main reasons why historical energy consumption may not be a good proxy for future energy consumption or future energy service level demand after electrification. These same two concepts could be applied to any other project type that provides services qualitatively and quantitatively different from historical service levels:

- Firstly, as incomes grow over time, energy service demand and consumption would increase, so that even without access to electricity it is likely that energy consumption in the 'without project scenario' would rise over time. This is the 'income effect'.

- Secondly, and more importantly, the combination of low household incomes and high unit costs of energy services can mean that individual households cannot afford sufficient energy services for their basic needs. In other words, since households face a budget constraint and must trade off purchasing energy services with other household needs, poor households may be forced to choose levels of energy services that are inadequate to meet their basic needs. If the households had access to a less expensive energy service (i.e. because of the availability of a less expensive source with lower unit cost, such as electric versus kerosene lighting), those households would consume significantly more energy services even without a change in total household income. This is the 'price effect' and it is due to a combination of lack of physical access to an energy source or technology (i.e. the lack of infrastructure barrier cited earlier) and a high unit cost of existing energy services. The price effect is similar to the 'rebound effect' described in the literature on energy efficiency (Greening et al., 2000; Lin et al., 2013).

Both the income effect and the price effect have been described as 'suppressed demand' for energy services that must be considered in setting the baseline for $\mathrm{CDM}$ projects that target energy services for poor communities (Gold Standard, 2011; Thorne et al., 2010; UNFCCC, 2011; Winkler and Thorne, 2002). The two concepts, however, have different implications for how to construct an alternative baseline scenario. In addition, the income effect is accepted as a way to adjust historical energy use to create a baseline, while the price effect has only recently been explicitly implemented in approved accounting methodologies under the $\mathrm{CDM}$. The implications for the baseline of these two components of suppressed demand are as follows:

- Baseline service level increase due to the 'income effect': If the main issue was the growth of energy consumption over time as incomes increase, and the costs of the energy service are fairly constant, then an appropriate baseline could start with historical energy consumption and increase this each year after project implementation, proportional to the increase in average household income in the target community (i.e. assuming an elasticity of demand for energy relative to income of 1). This is similar to the concept of a 'trend-adjusted' energy use projection in approved small-scale methodology 'AMS I.A Electricity generation by the user'. It could also include, for example, the household investing in more-efficient lighting technologies, owing to increased access to capital (e.g. kerosene pressure lamps, which are more expensive than hurricane lamps and therefore rarely used by the poorest communities).

- Baseline service level increase due to the "price effect': The second concept, whereby historically households have not been able to purchase adequate levels of energy services, implies that a change in the unit cost of those services (e.g. $\$$ /lumen-hour, not $\$ / \mathrm{kWh}$ ) could lead to an immediate and significant increase in energy service demand. Switching from kerosene lighting to electric lighting, for example, can reduce the unit cost of lighting by $90 \%$ or more and consumption of lighting services (lumens) may 
jump by a factor of 40 (ESMAP, 2002; IEG, 2008).

The CDM Gold Standard, as part of the development of an improved cook-stove methodology, has considered the concept of suppressed demand: 'Where a group of people are deprived of a reasonable level of human development in comparison to their peers, and the opportunity to achieve a satisfactory level of service is available through carbon financing calculated from the baseline level of service of their peers or from the project level of service achievable, then the appropriate adjustment to the baseline can be made' (Gold Standard, 2011). This is an example of the price effect. The Gold Standard biogas-digester methodology has a similar definition of suppressed demand to the one provided here.

'Satisfied demand' is the level of energy services that would be reached with access to better quality and more affordable services and increases in income. In other words, satisfied demand is the level of energy service demand of households in a given area when the income effect and the price effect have been overcome. A CDM activity may overcome the 'price effect' by introducing a technology that dramatically reduces the unit cost of an energy service (e.g. CFLs with grid electricity). While a CDM project will not overcome the income effect directly, this is most likely much smaller and also has already been considered a reasonable adjustment to make to the baseline in cases such as the methodology for electricity generation by the user mentioned above.

Another way of understanding the relationship between these concepts is the following: the reason historical energy service levels are not a good proxy for the 'without project' baseline is that 'satisfied demand' for those services has been 'suppressed' by both an 'income effect' and a 'price effect'. The difference between satisfied demand and the actual observed level of demand is the sum of these two effects. This means that there are multiple options for setting a baseline, depending on the degree to which suppressed demand is considered. In addition, another option for the baseline is a 'minimum service level', which would reflect the minimum necessary to provide for adequate, basic human needs.

The concept of a minimum service level is common not only in the energy sector (see review of targets and measures in Bazilian et al., 2010) but across many sectors, from the Millennium Development Goals (MDGs), to proposals for minimum levels of clean water per household, adequate household comfort levels (related to space heating and cooling), adequate nutritional levels and even 'threshold' poverty levels that represent a household's ability to achieve MDG service levels (Baer et al., 2008; Falkenmark, 1989; Howard and Bartram, 2003; Modi et al., 2006; Thorne et al., 2010). This concept is also included in the EB-approve 'Guidelines on the consideration of suppressed demand in CDM methodologies' (UNFCCC, 2012, 2011).

Figure 1 illustrates the service levels of these different baseline concepts, showing how the suppressed demand effects discussed above and minimum service level compared with historical service levels. The 'income effect' line simply shows the gradual expected increase in service demand resulting from increasing household income within the affected communities (assuming per capita income is growing, which is not true for all developing countries). In other words, it is the service level without the income effect suppressing that demand. The 'income and price effect' line shows the step change in service levels that would occur if the services were suddenly as inexpensive (per unit) as they will be once the project activity has been implemented. For example, in the Philippines the estimated cost of lighting with kerosene is 36 US cents per kilolumen-hour (klmh), while the cost of lighting with grid electricity is 0.75 US cents per klmh (ESMAP, 2002). If the household had been able to pay 0.75 US cents per klmh for kerosene lighting historically, they would have consumed far more lighting services. ${ }^{2}$ This is why the 'income and price effect' line has a significantly higher service level than historical consumption. Note that this line still slopes upwards, as household incomes grow. The 'satisfied demand' line is the level of services at which the entire income and price effect has been removed over a longer time period (e.g. 10 years). The 'minimum service level' line is flat because it reflects a standard 'adequate' 3 demand for basic services for a typical rural community, rather than being based on the current income of the households in that community. The minimum service level is lower than satisfied demand because most households would want more than the most basic services if they could afford it.

Figure 2 illustrates how project service levels could compare to the baseline scenario alternatives. Project service levels are much higher than historical service levels because of the impact that the $\mathrm{CDM}$ project has on the unit cost of services. Service levels could reach the minimum service level almost immediately owing to dramatically lower unit costs, but may still take time to reach satisfied demand because of the income level of the households or other specific characteristics of the community (e.g. energy taxes and subsidies, or appliance prices).

Energy consumption (GJ or kWh, as opposed to service level) in each scenario depends on the technology used to provide the service. For example, providing the minimum service level with the proj- 


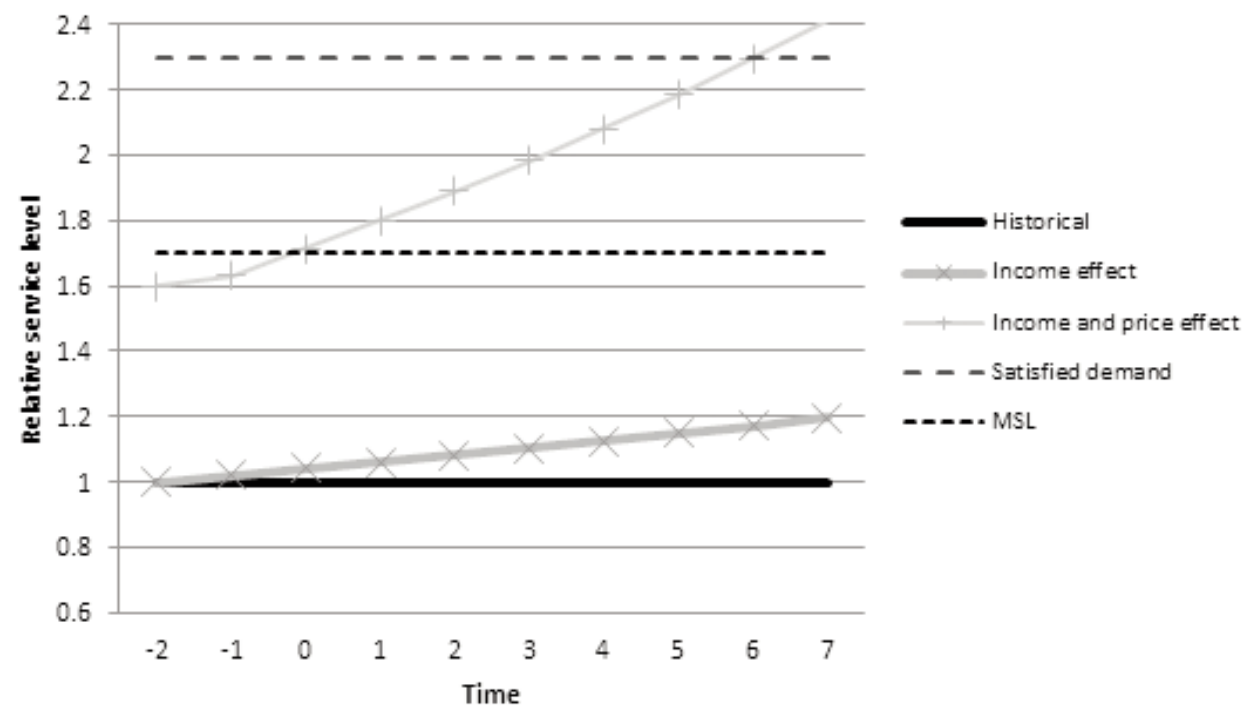

Note: Time is relative to the start of a CDM project activity. MSL = minimum service level.

Figure 1: Relative service levels for different baseline assumptions about suppressed demand effects and minimum service level

Source: Author's analysis

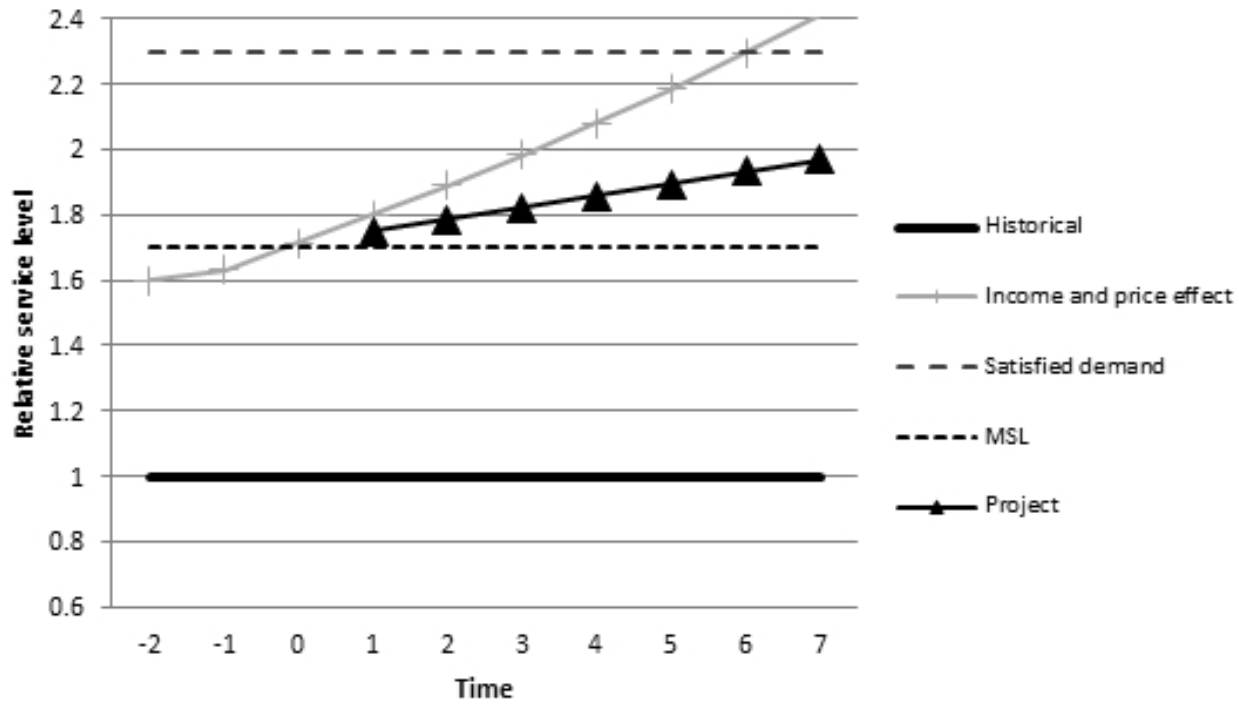

Note: Time is relative to start of CDM project activity. MSL = minimum service level. Income effect line has been removed for simplification.

Figure 2: Baseline and project service levels under different assumptions of suppressed demand effects and minimum service level

Source: Author's analysis

ect technology would use less energy than providing the same service with the historical technology. The project scenario might provide several times the service level of historical technology, but use less energy - in fact, this is what we would expect. Over time, however, the project scenario might use more energy than the household historically used, because the service level delivery is so much higher. This is particularly true if the project activity is grid electricity from a fossil fuel-based electricity grid. Figure 3 illustrates this possibility. The energy consumption of the baseline scenario alternatives in Figure 3 is shown using historical technology and is so marked 'hist', while the project scenario obviously uses the project technology. This shows how the project technology can meet the minimum service level and still be below historical energy consumption, because the project technology is so much more efficient at meeting the minimum service level. Of course, this figure does not provide an exact representation of these levels for all projects, but rather shows the possible relative differences. The relative emission levels would follow a similar pattern to the energy consumption levels, but obviously adjusted for the emission intensity of the fuels. The calculation of CERs would be very different if 


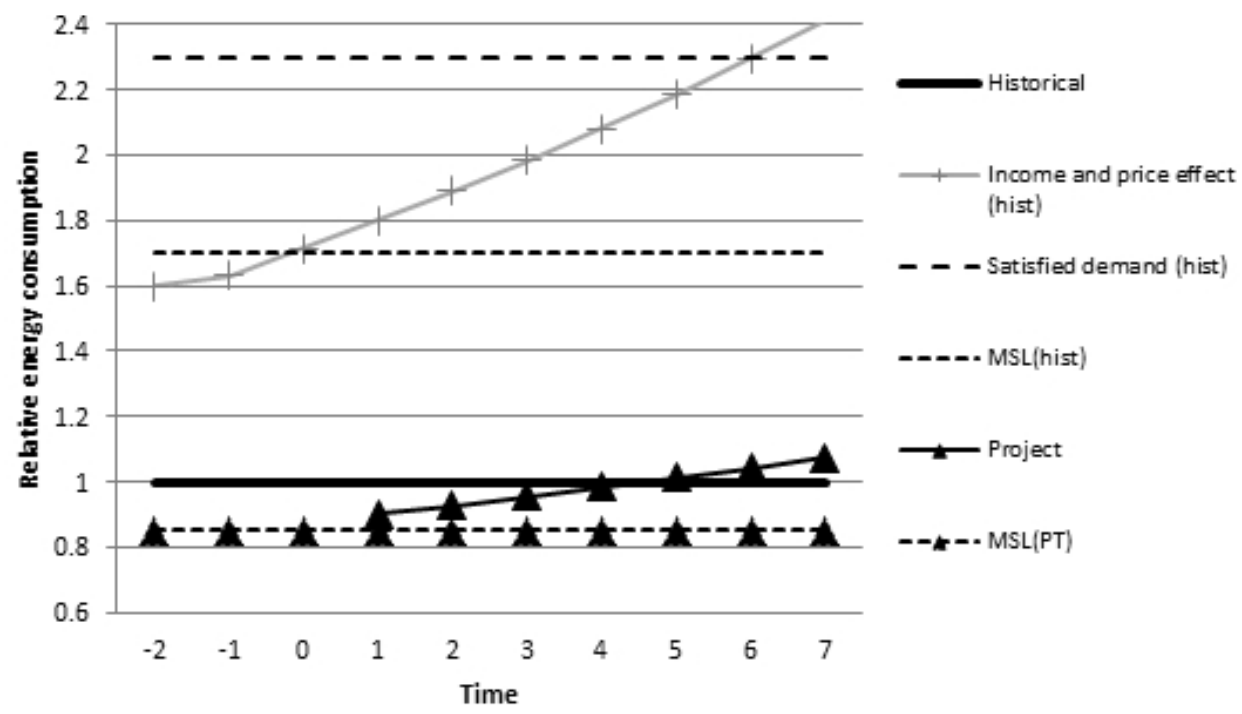

Note: The energy consumption levels are marked with (hist) to indicate the efficiency of the historical technology. For example, the minimum service level can be provided at a much lower energy consumption using project technology (PT). This implicitly assumes that the project technology is much more efficient than baseline technologies.

\section{Figure 3: Baseline and project energy consumption under different scenarios (historical versus} project technology)

Source: Author's analysis

comparing the project with the minimum service level using historical technology, instead of using actual historical energy consumption to calculate baseline emissions.

\section{Suppressed demand under the CDM}

Suppressed demand has been implicitly considered in some methodologies for greenfield projects, which do not use any historical consumption or production levels for the baseline. For example, ACM2 'Consolidated baseline methodology for grid-connected electricity generation from renewable sources' and AMS I.D 'Grid-connected renewable electricity generation', the most widely used methodologies, do not require the project participant to demonstrate that the CDM plant actually displaces another plant or that production is reduced elsewhere. The renewable power plant is essentially compared to the mix of power plants that would have been run or built to meet additional demand that exists but is currently not met owing to supply constraints. In the case of a geothermal or low power density hydropower plant (which both have some project emissions), this could mean that total emissions from the electricity grid actually increase as a result of the project, even though the emission intensity of electricity generation declines. This is reasonable, given the continuous and rapid growth in demand for electricity in almost all developing countries, and because of the large development benefits from increased access to and consumption of electricity.

Historically, this approach has not been applied to household energy services. Until recently, in the only methodology for household-scale renewable electricity provision, historical energy use was one of the baseline options, with the others being a diesel generator producing the same electricity or the electricity consumption in the closest grid-connected communities. While the last option could have addressed suppressed demand, the cost of monitoring was prohibitive for a small project. The diesel generator option was also problematic because the default emission factor used in the methodology was for a $200 \mathrm{~kW}$ capacity generator or larger. This implied that the most realistic alternative to household-scale renewable electrification was a local diesel mini-grid, when the capital cost for the latter would clearly be beyond the reach of that community even if the demand for energy services were there.

New methodologies and approaches are now emerging, along with important guidelines from the $\mathrm{EB}$, to address suppressed demand. In early 2011 a methodology for water purification was approved (AMS II.AV) which used project water-consumption levels to determine baseline emissions, even if the households did not boil that much water historically owing to lack of time and income. For rural electrification, AMS I.L (for household-scale off grid) and AMS III.BB (for grid connection) approved in 2012 both explicitly define a minimum service level and baseline technology for household lighting and household total electricity use and use a default baseline emission factor to represent that minimum service level. Baseline emissions therefore do not require monitoring, which simplifies the monitoring process considerably.

In July 2011 the EB approved guidelines on the treatment of suppressed demand in CDM method- 
ologies (UNFCCC, 2011e), and these were revised in July 2012 following inputs from stakeholders (UNFCCC, 2012). The guidelines provide methodological approaches for identifying the baseline technology/measure where there is suppressed demand and identifying the baseline service level used to calculate baseline emissions. The EB defined the minimum service level as, 'the service level that is able to meet basic human needs...basic housing, basic energy services including lighting, cooking, drinking water supply.... In some situations, this service level may not have been provided prior to the implementation of the CDM project activity'. The guidelines apply to any situation, 'when a minimum service level, as defined above, was unavailable to the end user of the service prior to the implementation of the project activity'. The guidelines outline the approach and principles for setting a minimum service level, 'that satisfies basic human needs and makes possible the development of the type of project'. The following guidance is also provided:

1. The minimum service level should be realistic and reasonable.

2. For establishing a minimum service level, the following approaches may be used:

(a) National/international peer-reviewed research or relevant studies (e.g. the World Health Organization recommendations on per capita safe drinking water);

(b) Benchmarks that take into account that emissions will rise to achieve the international/national development goals.

3. Further, in setting the minimum service level, the following should be taken into account:

(a) Environmental integrity of the emission reductions has to be safeguarded;

(b) Climatic zones may be taken into account where feasible;

(c) Normative decisions have to be clearly referenced and explained;

(d) Decisions regarding suppressed demand have to be re-evaluated and updated periodically based on recent data to ensure they are based on realistic assumptions.

The EB developed a work programme on suppressed demand, which included identifying methodologies for revision, public and expert consultation, revising methodologies and revising the guidelines themselves (UNFCCC, 2011i). The criteria for choosing these methodologies to revise included the opportunity to enhance the regional distribution of CDM projects, the wide use of the methodology by communities, whether minimum service levels can be considered in that technology area, and the exclusion of methodologies addressing industrial gases, processes and large-scale grid power generation (UNFCCC, 2012a).
Table 1 overleaf shows the methodologies where suppressed demand has been explicitly considered, in three different categories. The first group is from the work plan contained in the $\mathrm{EB}$ report, where the EB had requested the Secretariat and relevant support panels to explore how to incorporate suppressed demand. The second group is methodology revisions where the proponent of the revision motivated the change based on the Suppressed Demand Guidance. The final group is new methodologies that were developed after the approvals of the Suppressed Demand guidance and incorporated those ideas, as documented in the UNFCCC Methodology Guidebook (UNFCCC, 2013). Of the original 10 methodologies in the EB work plan, 5 were revised or replaced, while an additional 8 methodologies fall into the second and third categories. While the proportion of project activities influenced by these methodologies is very small, a significant share of CDM Programmes of Activities (PoAs) are utilising the revised or new methodologies.

\section{Implications and concerns}

The main concern raised by stakeholders regarding accounting for suppressed demand is that increasing baseline emission levels beyond historical emission levels could inflate the number of CERs issued and therefore undermine the environmental integrity of the CDM (e.g. Kollmuss, 2012). This concern does not imply that the household-level projects are not important, or that they are currently treated fairly, but that perhaps the CDM is not the best mechanism to address these project types. The current discussions around Results Based Financing (RBF) for mitigation, including the establishment of the Climate Initiative for Development (Ci-DEV) (www.ci-dev.org/) to address energy access issues, are looking at how payment levels could be increased even without changing the accounting for GHG impacts (ESMAP 2013).

The risks of increased crediting depends on the rate at which project beneficiaries would increase their emissions without the project, and how the minimum service level or other benchmark service level is set for that technology. If minimum service levels are set at levels that are achievable within a reasonable time frame (e.g. five to 10 years), this reduces the risk of inflating CER issuance. On the other hand, stakeholders have not criticised the greenfield power methodologies mentioned earlier for allowing crediting without actually displacing other power generation (current or future) and these are the most widely used CDM methodologies. There is an underlying equity issue here and a need for consistency across sectors and methodologies.

A related concern is that, even if the minimum service level is agreed, the choice of baseline tech- 
Table 1: Methodologies explicitly addressing suppressed demand or part of EB work plan on suppressed demand

Source: Fenhann (2014)

\begin{tabular}{|c|c|c|c|c|c|}
\hline \multirow{2}{*}{ Meth no. } & \multirow[b]{2}{*}{ Meth name } & \multirow[b]{2}{*}{ Revised } & \multirow[b]{2}{*}{ When } & \multicolumn{2}{|c|}{ Pipeline } \\
\hline & & & & Projects & PoAs \\
\hline & \multicolumn{5}{|l|}{ From EB67 work plan List of Meths } \\
\hline AM0025 & Alternative waste treatment processes & ACM22 & EB69 & 126 & 5 \\
\hline AM0046 & Distribution of efficient light bulbs to households & No & & 2 & 0 \\
\hline AM0086 & Installation of zero energy water purifier for safe drinking water application & No & EB70 & 1 & 0 \\
\hline AM0094 & Distribution of biomass based stove and/or heater for household or institution & No & EB70 & 0 & 0 \\
\hline$\overline{\mathrm{ACM}} 0014$ & Treatment of wastewater & Yes & EB77 & 47 & 1 \\
\hline$\overline{\mathrm{ACM}} 0016$ & Mass Rapid Transit Projects & No & & 15 & 1 \\
\hline AMS I.A & Electricity generation by the user & Yes & EB69 & 50 & 17 \\
\hline AMS I.E & Switch from non-renewable biomass for thermal applications by the user & Not nec. & EB70 & 27 & 58 \\
\hline AMS II.E & Energy efficiency and fuel switching measures for buildings & No & & 44 & 5 \\
\hline \multirow[t]{2}{*}{ AMS III.AR } & Substituting fossil fuel based lighting with LED/CFL lighting systems & Yes & EB68 & 4 & 14 \\
\hline & \multicolumn{5}{|l|}{ Additional revisions referring to Suppressed Demand } \\
\hline AM0091 & Energy efficiency technologies and fuel switching in new and existing buildings & Yes & EB77 & 0 & 0 \\
\hline AMS II.G & Energy efficiency measures in thermal applications of non-renewable biomass & Yes & EB70 & 45 & 68 \\
\hline \multirow[t]{2}{*}{ AMS III.F } & Avoidance of methane emissions through composting & Yes & EB67 & 103 & 21 \\
\hline & \multicolumn{5}{|l|}{ New methodologies where EB noted Suppressed Demand } \\
\hline $\mathrm{ACM} 0022$ & Alternative waste treatment processes & New & EB69 & 11 & \\
\hline AMS II.R & Energy efficiency space heating measures for residential buildings & New & EB73 & 0 & 0 \\
\hline AMS I.L & Electrification of rural communities using renewable energy & New & EB66 & 0 & 2 \\
\hline AMS III.BB & Electrification of communities through grid extension or new mini-grids & New & EB67 & 0 & 0 \\
\hline AMS III.AV & Low greenhouse gas emitting safe drinking water production systems & New & $\mathrm{EB} 60 / 62$ & 0 & 11 \\
\hline \multicolumn{4}{|c|}{ Total with revisions or new related to suppressed demand } & 413 & 197 \\
\hline \multicolumn{4}{|c|}{ Total pipeline } & 12011 & $452^{*}$ \\
\hline
\end{tabular}

nology has a dramatic influence on the baseline emissions. For example, the small-scale water purification methodology (AMS III.AR) allows the project participants to use boiling with non-renewable biomass as the baseline technology, even though many households may use low or non-energy intensive solutions (e.g. chlorination).

While some case studies are available on specific project types and how the concept of suppressed demand might be applied (GERES \& CDC Climat, 2011; Thorne, 2012), there is no research yet on the overall impact on carbon credit generation or emission reductions that could come from including suppressed demand in baselines. The methodologies prioritized for revision represent only $4 \%$ of the CDM pipeline so far, and $13 \%$ of the PoA pipeline, so clearly there is scope to expand their use. While there may be some tension between increasing the CDM's contribution to sustainable development and ensuring that emission reductions are 'real' and 'measurable', recognizing the future growth in emissions and incorporating that into baselines is clear- ly a priority for the Parties to the Kyoto Protocol given the decision at the Copenhagen Conference that initiated the EB's effort. So in this sense, this may be more a political decision than a technical or scientific decision.

A final concern is how to establish the minimum service levels in a way that is 'realistic and reasonable but not overly conservative'. This may require not only substantial expert input but also more stakeholder consultation than methodologies typically have received in the past. Because setting minimum service levels is a normative decision and involves expert judgement, the process must be transparent and thorough. This is similar to the challenge faced in setting standardized baselines.

\section{Conclusions}

The concept of suppressed demand, although often mentioned in the discussions about the CDM, should be defined more clearly, to distinguish between 'income effects' and 'price effects'. This would guide further methodological development 
in a way that could promote the more rapid uptake of clean energy technologies in poor communities as CDM projects. The guidance provided by the $\mathrm{EB}$, and the subsequent revisions of methodologies and newly approved methodologies, is important steps forward. Further guidance is needed, however, on several key issues. These include the following:

- Limiting methodological changes to account for suppressed demand to the technologies and sectors that directly address household-level services.

- Developing a clear plan for approving 'minimum service levels' and baseline technology choices, including which stakeholders and experts will be involved and how.

- Providing guidance on the time frame within which the 'minimum service levels' should be achievable.

- Ensuring that the 'minimum service levels' are universal and not country specific.

- Using the methodology revision process to establish consistency across all sectors.

- Providing guidance on how often the 'minimum service level' and/or baseline technology should be reviewed and, if necessary, updated.

\section{Notes}

1. While the concept of suppressed demand could be applied to other sectors, such as industrial and commercial energy use, these are conceptually different for two reasons: the budget constraint is related access to capital issues rather than to poverty, and the difference in the price of energy service options available tends to be much smaller than for households (e.g. consider different commercial lighting options, as opposed to a household situation where kerosene wick lamps up to high efficiency bulbs are all options).

2. The difference in GHG-related external costs between these two services depends on both the delivery technology and the service level. Lighting with a $60 \mathrm{~W}$ incandescent and a kerosene hurricane lamp, for example, might have fairly similar emissions over the course of the year, but the incandescent bulb would provide almost 20 times the service level (e.g. luxhours) even when use the same number of hours.

3 The Guidelines provide different options for justifying the adequacy of the proposed service level, including: (1) The service level provided prior to the implementation of the project activity, (2) The service level provided under the project activity, and (3) A minimum service level derived from 'globally applicable conservative thresholds.' The latter may be based on, for example, 'national/international peer-reviewed research or relevant studies,' or, 'benchmarks that take into account that emissions will rise to achieve the international/national development goals.'

\section{References}

Baer, P., Athanasiou, T., Kartha, S., and Kemp-Benedict, E., (2008). The Greenhouse Development Rights Framework. The right to development in a climate constrained world (No. Second edition). Heinrich Böll Foundation, Christian Aid, EcoEquity and the Stockholm Environment Institute, Berlin.

Bazilian, M., Nussbaumer, P., Cabraal, A., Centurelli, R., Detchon, R., Gielen, D., Rogner, H., Howells, M., McMahon, H., Modi, V., Nakicenovic, N. O'Gallachoir, B., Radka, M., Rijal, K., Takada, M., and Ziegler, F., (2010). Measuring energy access: Supporting a global target. Earth Institute, Columbia University, New York.

Ellis, J., Corfee-Morlot, J., and Winkler, H., (2007). CDM: Taking stock and looking forward. Energy Policy 35, 15-28.

ESMAP, (2002). Rural Electrification and Development in the Philippines: Measuring the Social and Economic Benefits. Energy Sector Management Assistance Programme, World Bank, Washington, D.C.

ESMAP, (2013). Results-Based Financing in the Energy Sector: An Analytical Guide. Energy Sector Management Assistance Programme, World Bank, Washington, D. C.

Falkenmark, M., (1989). The massive water scarcity threatening Africa-why isn't it being addressed. Ambio 18, 112-118.

Fenhann, J., (2014). UNEP Risø CDM/JI Pipeline Analysis and Database, 1 February 2014 [WWW Document]. URL www.cdmpipeline.org (accessed 12.5.13).

GERES \& CDC Climat, (2011). Three case studies of suppressed demand in CDM projects: case studies on development projects in the carbon market. GERES and CDC Climat, Aubange, France.

Gillenwater, M., and Seres, S., (2011). The Clean Development Mechanism: a review of the first international offset programme. Greenh. Gas Meas. Manag. 1, 179-203. doi:10.1080/20430779.2011.647014.

Gold Standard, (2011). Technologies and Practices to Displace Decentralized Thermal Energy Consumption [WWW Document]. URL http://www.cdmgoldstandard.org/wpcontent/uploads/2011/10/GS 110411 TPDDTEC M ethodology.pdf. (accessed 7.3.12).

Greening, L.A., Greene, D.L., and Difiglio, C., (2000). Energy efficiency and consumption - the rebound effect - a survey. Energy Policy 28, 389-401.

Howard, G., and Bartram, J., (2003). Domestic Water Quantity, Service, Level and Health. World Health Organisation, Geneva.

IEG, (2008). The welfare impacts of rural electrification: a reassessment of the costs and benefits. An IEG impact evaluation. Independent Evaluation Group, World Bank, Washington, D. C.

Kollmuss, A., (2012). Suppressed Demand. An NGO Perspective. Presented at the UNFCCC Sustainable Development Methodologies Workshop, CDM Watch, Bonn.

Lin, B., Yang, F., and Liu, X., (2013). A study of the 
rebound effect on China's current energy conservation and emissions reduction: Measures and policy choices. Energy 58, 330-339. doi:10.1016/j.energy.2013.06.018.

Modi, V., McDade, S., Lamment, D., and Saghir, J., (2006). Energy Services for the Millennium Development Goals (No. Millennium Project). Energy Sector Management Assistance Programme, United Nations Development Programme, UN Millennium Project, and World Bank, New York.

Schneider, L., (2009). Assessing the additionality of CDM projects: practical experiences and lessons learned. Clim. Policy 9, 242-254. doi:10.3763/cpol.2008.0533.

Sovacool, B.K., (2011). Conceptualizing urban household energy use: Climbing the 'Energy Services Ladder'. Energy Policy 39, 1659-1668. doi:10.1016/j.enpol.2010.12.041.

Thorne, S., (2012). Operationalising Suppressed Demand. Presentation to First Sustainable Development Mechanisms Joint Workshop. 24 March 2012 [WWW Document]. URL http://cdm.unfccc.int/stakeholder/workshops/jws/sdm jws/presentations/jws_pres32.ppt (accessed 6.21.12).

Thorne, S., Mqadi, L., Winkler, H., Wamukonya, N., Michaelowa, A., and La Rovere, E.L., (2010). Energy poverty and suppressed demand for energy services - case study of low cost housing in South Africa. Unpublished working draft. SouthSouthNorth Project, Cape Town.

UNFCCC, (2006). Decision 3/CMP.1 Modalities and procedures for a clean development mechanism as defined in Article 12 of the Kyoto Protocol. FCCC/KP/CMP/2005/8/Add.1 [WWW Document]. URL http://cdm.unfccc.int/Reference/COPMOP/ 08a01.pdf (accessed 6.21.12).

UNFCCC, (2009). Decision 2/CMP.5. Further guidance relating to the clean development mechanism. FCCC/KP/CMP/2009/21/Add.1. United Nations Framework Convention on Climate Change.

UNFCCC, (2011). Guidelines on the consideration of suppressed demand in CDM methodologies (version 01). EB62 Report. Annex 6. United Nations Framework Convention on Climate Change, Bonn.

UNFCCC, (2012). Guidelines on the consideration of suppressed demand in CDM methodologies (version 02). EB68 Report. Annex 2. United Nations Framework Convention on Climate Change, Bonn.

UNFCCC, (2013). CDM Methodology Booklet. Information up to EB75 November 2013. United Nations Framework Convention on Climate Change, Bonn.

Winkler, H., and Thorne, S., (2002). Baselines for suppressed demand: CDM projects contribution to poverty alleviation. South Afr. J. Econ. Manag. Sci. 5, 413-429. 